\title{
Avaliação do perfil cromatográfico obtidos por CLAE-DAD e da atividade antioxidante das folhas de espécies Campomanesia sessiliflora (O. Berg) Mattos e Campomanesia xanthocarpa O. Berg
}

\author{
KATAOKA, V.M.F.; CARDOSO, C.A.L.* \\ Universidade Estadual de Mato Grosso do Sul, Curso de Química, Rodovia Dourados/Itahun Km 12, Caixa \\ Postal 351, CEP: 79804-970, Dourados-Brasil. *claudia@uems.br
}

\begin{abstract}
RESUMO: O objetivo desta pesquisa visou a avaliação do perfil cromatográfico, a atividade antioxidante, e os teores de fenóis e flavonóides nos extratos orgânicos e infusos de folhas das espécies $C$. xanthocarpa e $C$. sessiliflora coletadas durante os períodos vegetativo e reprodutivo. As análises dos extratos e infusos foram realizadas empregando-se cromatografia líquida de alta eficiência com detector de arranjo de diodos (CLAE-DAD). Segundo a comparação dos perfis obtidos foi baixa a similaridade entre a composição química das duas espécies. O teor de fenóis $\left(64,69-334,18 \mu \mathrm{g}\right.$ de ácido gálico $\left.\mathrm{mL}^{-1}\right)$ foi superior ao teor de flavonóides $(4,02-$ $35,60 \mu \mathrm{g}$ de quercetina $\mathrm{mL}^{-1}$ ) em todas as análises. Os extratos orgânicos e infusos das folhas coletadas no período reprodutivo apresentaram teores de fenóis e de flavonóides superiores em ambas as espécies. Não houve uma correlação linear entre o teor de fenóis e flavonóides com a atividade antioxidante em todas as amostras.
\end{abstract}

Palavras-chave: Campomanesia sessiliflora, Campomanesia xanthocarpa, CLAE-DAD, guavira, guabiroba

\begin{abstract}
Evaluation of the chromatographic profile and the antioxidant activity of the species Campomanesia sessiliflora (O. Berg) Mattos and Campomanesia xanthocarpa O. Berg. The aim of this study was to evaluate the chromatographic profile, the antioxidant activity, and the levels of phenols and flavonoids in organic extracts and infusions from leaves of the species $C$. xanthocarpa and $C$. sessiliflora collected during the vegetative and reproductive periods. Analyses of the extracts and infusions were performed by adopting high-performance liquid chromatography with diode array detector (HPLC-DAD). Based on the comparison of the obtained profiles, similarity between the chemical composition of the two species was low. Phenol levels (64.69-334.18 $\mu$ gallic acid $\mathrm{mL}^{-1}$ ) were superior to the level of flavonoids (4.02$35.60 \mu \mathrm{g}$ quercetin $\mathrm{mL}^{-1}$ ) in all analyses. Organic extracts and infusions from leaves collected in the reproductive period had superior phenol and flavonoid levels in both species. There was not a linear correlation of phenol and flavonoid levels with antioxidant activity in all samples.
\end{abstract}

Key words: Campomanesia sessiliflora, Campomanesia xanthocarpa, HPLC-DAD, "guavira", "guabiroba"

\section{INTRODUÇÃO}

A Campomanesia sessiliflora (O. Berg) é denominada guabirobeira-verde encontrada em cerrados e campos do Brasil Central, Minas Gerais e Mato Grosso do Sul. A Campomanesia xantocarpa $O$. Berg é encontrada facilmente no habitat natural desde Minas Gerais e Mato Grosso do Sul até Rio Grande do Sul. Ambas apresentam frutos de polpa suculenta, firme e de sabor doce. Estas espécies são distinguidas pelo aspecto das folhas e flores (Lorenzi et al., 2006).
No Sul do Brasil, C. xanthocarpa, tem sido empregada empiricamente, pelo seu efeito potencial na redução de colesterol no sangue (Klafke et al., 2010).

Estudos químicos por cromatografia líquida de alta eficiência (CLAE), nas folhas relataram à presença de quercetina, miricitrina e rutina em Campomanesia xanthocarpa; miricitrina em Campomanesia pubences e miricitrina e quercetina em Campomanesia quazumaefolia (Schmeda-

Recebido para publicação em 07/04/2010

Aceito para publicação em 06/09/2012

Rev. Bras. PI. Med., Botucatu, v.15, n.1, p.121-129, 2013. 
Hirschmann, 1995).

Em Campomanesia xanthocarpa foi realizada análise química, utilizando CLAE e identificou flavonóides, saponinas e taninos, também foram realizados testes de atividade antiulcera e de toxicidade em ratos (Markman, 2002; Markman et al., 2004).

Tratamento com infusão das folhas de Campomanesia xanthocarpa em ratos com dieta de alta caloria, mostrou redução significante em ganho de peso e diminuição da glicemia, não se observou redução significativa de triglicérideos e colesterol em comparação ao grupo de controle (Biavatti et al., 2004)

Estudos preliminares com extratos das folhas de C. xanthocarpa indicaram que estas reduzem as taxas de colesterol (Klafke et al.; 2008).

As capsulas preparadas com folhas de C. xanthocarpa apresentaram uma diminuição significativa nos níveis de coleserol total e LDL em pacientes que consumiram as mesmas (Klafke et al., 2010).

Estudos sugerem que o tratamento com o decocto das folhas de $C$. xanthocarpa $(20 \mathrm{~g} / \mathrm{L})$ possa ser útil para o manejo do diabete melito, porém estudos farmacológicos e toxicológicos ainda são necessários (Vinagre et al., 2010).

Altos teores de ácidos urônicos, arabinose e galactose detectados em sua polpa, indicam que esta é constituída por polissacarídeos pécticos. Os polissacarídeos extraídos da polpa da gabiroba apresentaram um comportamento pseudoplástico (Santos et al., 2010).

Estudos com gêneros da família Myrtaceae revelaram que a espécie $C$.xanthocarpa apresentou os sesquiterpenos como componentes majoritários no óleo essencial de folhas (Menut et al., 1996; Limberger et al., 2001).

Os óleos essenciais extraídos das folhas de C. xanthocarpa revelaram atividade antimicrobiana em relação ao Staphylococcus aureus, Salmonella cholerasuis e Candida albicans (Markman et al., 2002).

Análise do óleo essencial dos frutos de C. xanthocarpa indicaram que o limoneno $(10,9 \%)$ e $\beta$-cariofileno $(21,8 \%)$ são seus componentes majoritários e que este óleo apresenta atividade antioxidante (Marin et al., 2008).

De acordo com Vallilo et al. (2008), os frutos de $C$. xanthocarpa apresentam alto teor de água, $1,9 \%$ de lipídeos, $8,9 \%$ de carboidratos totais, $6,3 \%$ de fibra alimentar e minerais como potássio, fósforo e magnésio além de microelementos. Também em seu óleo essencial foram identificados 62 componentes, sendo os majoritários $\alpha$-pineno (15\%) o-cimeno (10,8\%), e $\beta$-pineno $(10,5 \%)$.

A polpa dos frutos maduros de $C$. xanthocarpa foi analisada por Santos et al. (2009), e apresentou $3,84 \%$ de fibra bruta, $0,12 \%$ de lipídeos e $1,08 \%$ de proteínas. O teor de compostos fenólicos foi significativo, assim como o de vitamina C $(233,56 \mathrm{mg} / 100 \mathrm{~g})$.

Os óleos essenciais obtidos a partir dos frutos de C. xanthocarpa e C. adamantium (Myrtaceae) foram analisados por CG e CG-EM, sendo que nas duas espécies o maior constituinte foi cryptomeridiol (11,9-19,2\%) (Cardoso et al., 2009).

Em estudos das sementes de C. xanthocarpa o extrato etanólico apresentou o maior potencial antioxidante, bem como a maior quantidade de compostos fenólicos totais. No óleo, destacou-se maior porcentagem de ácidos graxos insaturados, sendo o principal componente o ácido oléico (Santos et al., 2012).

Foram identificadas as substâncias majoritárias (E)-salveno, (Z)- $\beta$-ocimeno, mirtenal, viridifloreno, $\alpha$-tujopsan-2-ol, leptospermona iso presentes no óleo essencial das folhas de Campomanesia sessiliflora utilizando cromatografia gasosa acoplada a espectrometria de massas (CGEM) (Gnoatto et al., 2010).

O presente trabalho teve como objetivo avaliar o perfil cromatográfico, a atividade antioxidante, os teores de fenóis e flavonóides dos extratos orgânicos e infusos das folhas das espécies C. xanthocarpa e C. sessiliflora coletadas durante o período vegetativo e reprodutivo.

\section{MATERIAL E MÉTODO}

\section{Plantas}

As folhas de C. xanthocarpa foram coletadas em Itaporã-MS (período vegetativo e reprodutivo) segundo as coordenadas geográficas: latitude $22^{\circ} 07^{\prime} 39,5^{\prime \prime}$ S e longitude $054^{\circ} 48^{\prime} 61$, 2" W. A espécie foi identificada e uma exsicata foi depositada no Herbário da UFGD, Dourados, Brasil, sob o número 1203.

As folhas de $C$. sessiliflora foram coletadas em Dourados-MS (período vegetativo e reprodutivo) segundo as coordenadas geográficas: latitude $22^{\circ}$ $11^{\prime}$ ' $81,9^{\prime \prime}$ S e longitude $054^{\circ} 55^{\prime} 81,2^{\prime \prime}$ W. A espécie foi identificada e uma exsicata foi depositada no Herbário da UFGD, Dourados, Brasil, sob o número 1204.

As folhas de C. xanthocarpa e C. sessiliflora foram trituradas em triturador de aço e para o preparo das soluções foi empregada a proporção de $50 \mathrm{mg}$ de folhas secas para $25 \mathrm{~mL}$ de solvente. Para o preparo do extrato hidroetanólico foi empregado etanol:água 80:20 v/v por 2 horas de extração em ultrassom e para o preparo do infuso foi empregada água quente $\left(95^{\circ} \mathrm{C}\right)$ abafado durante 30 minutos.

Rev. Bras. PI. Med., Botucatu, v.15, n.1, p.121-129, 2013. 
Após a extração as amostras foram filtradas com papel filtro e reconstituídas em balão volumétrico de mesmo volume da quantidade do solvente extrator empregado (Tabela 1).

\section{Análises por CLAE-DAD}

As análises foram realizadas empregando cromatógrafo líquido de alta eficiência modelo: Varian 210, detector de arranjo de diodos (DAD), com varredura entre $200-800 \mathrm{~nm}$. Coluna de fase reversa C-18 ( $25 \mathrm{~cm} \times 4,6 \mathrm{~mm} \times 5 \mu \mathrm{m})$ e pré-coluna $(2,5 \mathrm{~cm} \times 3 \mathrm{~mm})$ de mesma fase da coluna. A eluição foi realizada em sistema ternário e gradiente, para ambas as espécies (Tabela 2). 0 tempo de análise foi de 50 minutos, a vazão de fluxo da bomba de 1 $\mathrm{mL} \mathrm{min}^{-1}$ e volume injetado de $50 \mu \mathrm{L}$.

\section{Análise empregando espectrofotometria de absorção molecular \\ Os testes de fenóis totais, flavonóides} e atividade antioxidante foram realizados em Espectrofotômetro 700S Femto (190-1100 nm). Os testes foram realizados em triplicata.

\section{Teste de fenóis totais}

A cada $100 \mu \mathrm{L}$ das amostras foram adicionados $1,5 \mathrm{~mL}$ de solução aquosa de carbonato de sódio $2 \%, 0,5 \mathrm{~mL}$ de reagente Folin-Ciocalteau $(10 \%)$ e $1 \mathrm{~mL}$ de água destilada, após 30 minutos efetuou-se a leitura em espectrofotômetro no comprimento de onda de $760 \mathrm{~nm}$. O mesmo procedimento foi realizado para o branco, sendo substituído $100 \mu \mathrm{L}$ de amostra por $100 \mu \mathrm{L}$ do solvente extrator (etanol:água $80: 20 \mathrm{v} / \mathrm{v}$ para o extrato hidroetanólico ou água para o infuso) (Djeridane et al., 2006).

Para calcular a concentração de fenóis totais foi preparada uma curva analítica empregando o ácido gálico $(2,1 ; 10,3 ; 20,6 ; 30,9 ; 41,2 ; 82,4$ e $123,6 \mu \mathrm{g})$ como padrão e as respectivas absorbâncias foram lidas. Através da regressão linear foi obtida a equação da reta, com a $=0,02443$ (coeficiente linear), $b=0,01266$ (coeficiente angular) e $r=$ 0,99941 (coeficiente de correlação). O procedimento experimental realizado com o padrão foi o mesmo utilizado para as amostras. O resultado foi expresso $\mathrm{mg}$ de ácido gálico por $\mathrm{mL}$ de extrato ou infuso.

\section{Teste de flavonóides}

A cada $500 \mu \mathrm{L}$ das amostras foram adicionados 1,5 mL de álcool etílico $95 \%, 0,1 \mathrm{~mL}$ de cloreto de alumínio $10 \%\left(\mathrm{AlCl}_{3} \cdot 6 \mathrm{H}_{2} \mathrm{O}\right), 0,1 \mathrm{~mL}$ de acetato de sódio $\left(\mathrm{NaC}_{2} \mathrm{H}_{3} \mathrm{O}_{2} \cdot 3 \mathrm{H}_{2} \mathrm{O}\right)\left(1 \mathrm{~mol} \mathrm{~L}^{-1}\right) \mathrm{e}$ $2,8 \mathrm{~mL}$ de água destilada após 40 minutos fez-se a leitura no espectrofotômetro num comprimento de onda de $415 \mathrm{~nm}$. O mesmo procedimento foi realizado para o branco, sendo substituído $500 \mu \mathrm{L}$ de amostra por $500 \mu \mathrm{L}$ de (etanol:água 80:20 v/v para o extrato hidroetanólico ou água para o infuso) (Lin \& Tang, 2007).

Para calcular a concentração de flavonóides foi preparada uma curva analítica empregando a quercetina $(2,5 ; 5,0 ; 10,0 ; 20,0 ; 25,0 ; 50,0$; 100,0 e $125,0 \mu \mathrm{g}$ ) como padrão e as respectivas absorbâncias foram lidas. Com estes dados foi feita a regressão linear e foi obtida a equação da reta, a qual teve os dados empregados no cálculo das amostras reais, $a=0,00246, b=0,01048$ e $r=0,99975$. $O$ resultado foi expresso em $\mu \mathrm{g}$ de quercetina por $\mathrm{mL}$ de extrato ou infuso. O procedimento experimental realizado com o padrão foi o mesmo utilizado para as amostras.

\section{Ensaio antioxidante com o radical livre DPPH}

$A$ atividade antioxidante das amostras, empregando o radical livre DPPH (1,1-difenil-2-picril-

TABELA 1. Descrição das amostras analisadas referente ao tipo de extrato, ao período e local de coleta para as folhas de C. xanthocarpa e C. sessiliflora.

\begin{tabular}{|c|c|c|c|c|c|c|}
\hline \multirow[t]{2}{*}{ Código } & \multirow[t]{2}{*}{ Espécie } & \multirow[t]{2}{*}{ Extrato } & \multicolumn{2}{|c|}{ Período } & \multicolumn{2}{|c|}{ Local de coleta } \\
\hline & & & $\mathbf{v}$ & $\mathbf{R}$ & 1 & D \\
\hline XE8S & C. xanthocarpa & Hidroetanolico & $x$ & & $x$ & \\
\hline RXE8S & C. xanthocarpa & Hidroetanolico & & $x$ & $x$ & \\
\hline SE8S & C. sessiliflora & Hidroetanolico & $x$ & & & $x$ \\
\hline RSE8S & C. sessiliflora & Hidroetanolico & & $x$ & & $x$ \\
\hline XIS & C. xanthocarpa & Infuso & $x$ & & $x$ & \\
\hline RXIS & C. xanthocarpa & Infuso & & $x$ & $x$ & \\
\hline SIS & C. sessiliflora & Infuso & $x$ & & & $x$ \\
\hline RSIS & C. sessiliflora & Infuso & & $x$ & & $x$ \\
\hline
\end{tabular}

V: Vegetativo; R: Reprodutivo; I: Itaporã; D: Dourados. 
TABELA 2. Método de eluição (sistema ternário e gradiente) por CLAE-DAD empregado para as análises das amostras do gênero Campomanesia.

\begin{tabular}{cccc}
\hline Tempo (min) & Metanol & Água & Acetonitrila \\
\hline 0 & $1 \%$ & $84 \%$ & $15 \%$ \\
15 & $1 \%$ & $59 \%$ & $40 \%$ \\
20 & $1 \%$ & $21 \%$ & $78 \%$ \\
30 & $1 \%$ & $0 \%$ & $99 \%$ \\
40 & $0 \%$ & $0 \%$ & $100 \%$ \\
50 & $1 \%$ & $84 \%$ & $15 \%$ \\
\hline
\end{tabular}

hidrazila) $0,004 \%$ em metanol, foi verificada para os volumes de 20,100 e $500 \mu \mathrm{L}$ das amostras, as quais foram diluídas para um volume final de $1 \mathrm{~mL}$. A cada $1 \mathrm{~mL}$ da solução preparada foram adicionados 2 $\mathrm{mL}$ da solução de DPPH, através das absorbâncias resultantes, foi calculado o percentual de inibição do radical livre DPPH. O comprimento de onda de leitura foi $517 \mathrm{~nm}$. O efeito "sequestro" de radical (\%) foi calculado usando a seguinte equação:

$$
\% \Delta_{0}=\frac{\left(A_{0}-A\right) \times 100}{A_{0}}
$$

Onde Ao é a absorbância do DPPH na mistura (solvente extrator+metanol) decorridos 30 minutos de reação, $A$ corresponde à absorbância da amostra em DPPH decorridos 30 minutos de reação (Kumaran \& Karunakaran, 2006).

\section{RESULTADO E DISCUSSÃO}

O perfil cromatográfico das folhas de $C$. xanthocarpa e C. sessiliflora foi comparado (Tabela 3 ) entre os extratos hidroetanólicos e infusos de folhas nos períodos vegetativo e reprodutivo empregando como parâmetros o tempo de retenção e o espectro de absorção molecular obtido através do detector de arranjo de diodos. Foram analisadas as amostras (XE8S, RXE8S, XIS, RXIS, SE8S, RSE8S, SIS e RSIS descritas na Tabela 1) e os picos que apresentaram similaridade com relação aos parâmetros monitorados para as duas espécies foram numerados. Não denominamos de substância, pois no pico pode haver mais de uma substância.

Analisando os dois períodos e as duas espécies (Figuras 1-8) pode-se constatar que 0 pico 1 está presente tanto nos infusos quanto nos extratos de $C$. sessiliflora em ambos os períodos. O pico 2 está presente nos extratos orgânicos das duas espécies tanto no período vegetativo quanto no período reprodutivo. Os picos 3 e 4 estão presentes nos extratos hidroetanólicos nos dois períodos em C. xanthocarpa, sendo que o pico 3 também está presente no infuso do período vegetativo. O pico 5 está presente nos extratos hidroetanolicos e infusos em $C$. xanthocarpa e C. sessiliflora. O pico 6 está presente no período reprodutivo em $C$. sessiliflora e no período vegetativo em $C$. xanthocarpa. O pico 7 está presente nos extratos hidroetanolicos do período vegetativo das duas espécies. Os outros picos presentes no cromatograma estão em apenas uma das amostras analisadas. Podemos verificar através das figuras 1-8 que o pico 5 é o único presente nas duas espécies e em todos os tipos de extratos, que o pico 1 é característico da espécie $C$. sessiliflora e que os picos 3 e 4 são característicos em C. xanthocarpa.

Os demais picos presentes nos cromatogramas, mesmo com tempos de retenção semelhantes não apresentaram similaridade quanto ao espectro de absorção molecular indicando que são substancias diferentes. Em todas as análises o tempo de retenção apresentou coeficiente de variação (CV) abaixo de $4,0 \%$ indicando boa reprodutibilidade das análises (Tabela 3 ).

Nas infusões (Figuras 3, 4, 7 e 8) é possível observar que não houve a extração de algumas

TABELA 3. Tempo de retenção dos picos monitorados presentes nas análises das amostras de C. xanthocarpa e C. sessiliflora.

\begin{tabular}{clccc}
\hline Pico & Amostras & $\lambda_{\text {Abs }}$ & TR \pm SD & CV $\%$ \\
\hline 1 & SE8S, RSE8S, SIS, RSIS & 205,$84 ; 274,46$ & $6,20 \pm 0,19$ & 3,06 \\
2 & XE8S, RXE8S, SE8S, RSE8S & 230,$42 ; 275,57 ; 330,07$ & $22,49 \pm 0,14$ & 0,62 \\
3 & XE8S, RXE8S, XIS & 209,$28 ; 286,87$ & $23,67 \pm 0,12$ & 0,51 \\
4 & XE8S, RXE8S & 211,$16 ; 290,46$ & $24,65 \pm 0,17$ & 0,69 \\
5 & XE8S, RXE8S, SE8S, RSE8S, XIS, RXIS, SIS, RSIS & 222 & $26,26 \pm 0,13$ & 0,50 \\
6 & XE8S, RSE8S & 277,59 & $26,97 \pm 0,02$ & 0,07 \\
7 & XE8S, SE8S & 202,$62 ; 282,54$ & $33,69 \pm 0,10$ & 0,30 \\
\hline
\end{tabular}

$\lambda_{\text {Abs }}$ : Bandas de absorção (nm); TR: Tempo de retenção (min); SD: Desvio padrão; CV: Coeficiente de variação (\%). 


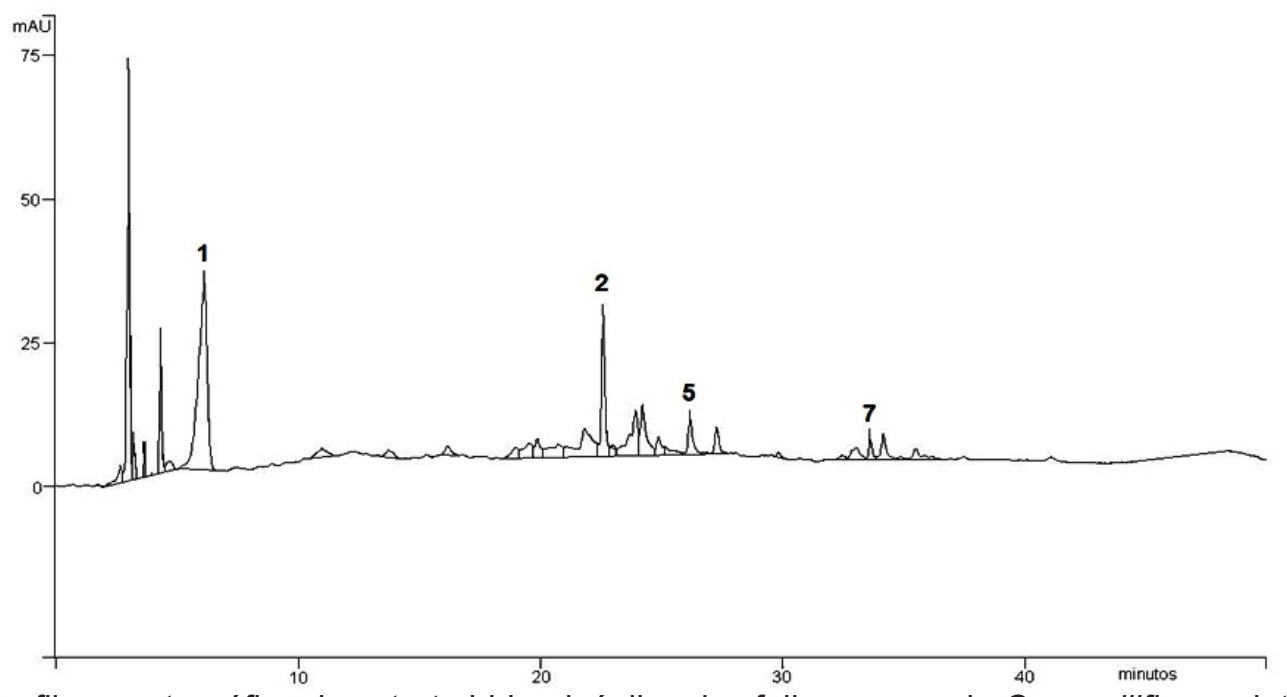

FIGURA 1. Perfil cromatográfico do extrato hidroalcóolico das folhas secas de $C$. sessiliflora coletadas durante o período vegetativo (SE8S). Condições cromatográficas vide Tabela 2.

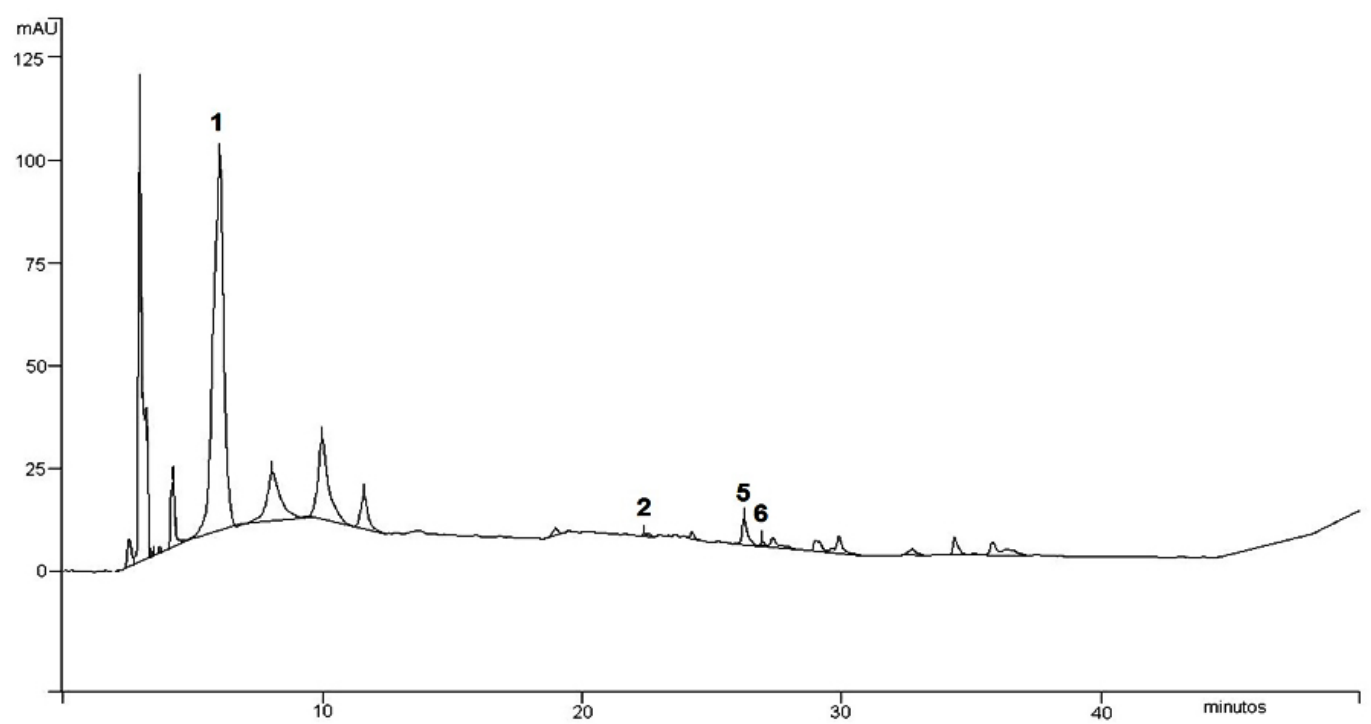

FIGURA 2. Perfil cromatográfico do extrato hidroalcóolico das folhas secas de $C$. sessiliflora coletadas durante o período reprodutivo (RSE8S). Condições cromatográficas vide Tabela 2.

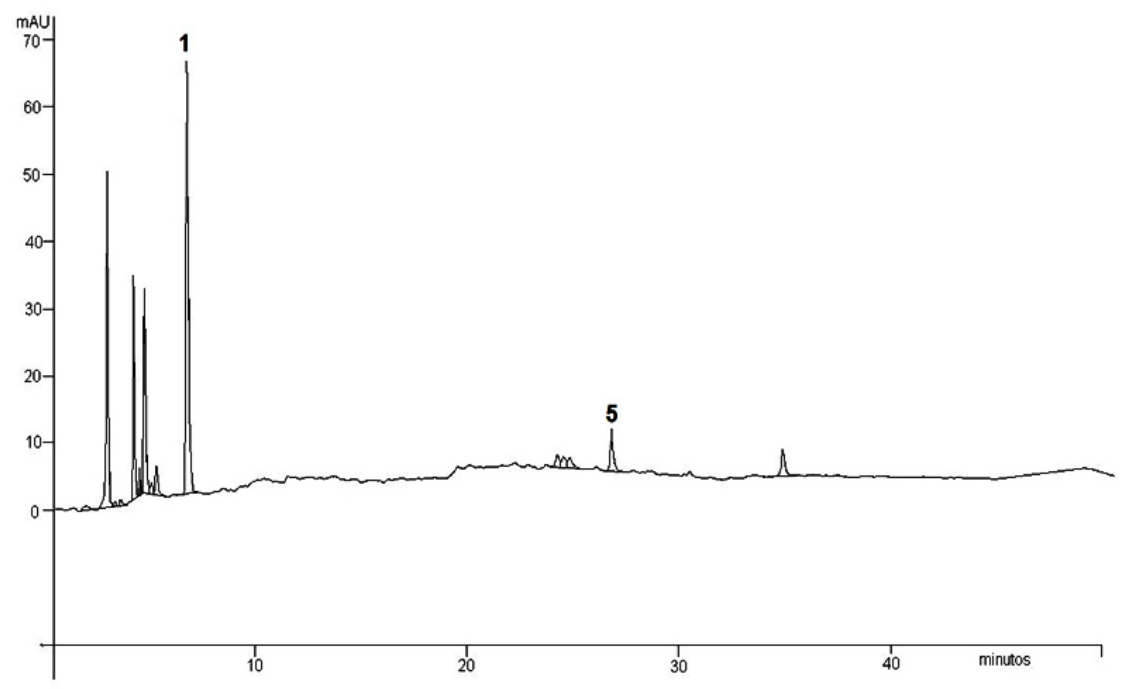

FIGURA 3. Perfil cromatográfico do extrato infuso das folhas secas de C. sessiliflora coletadas durante o período vegetativo (SIS). Condições cromatográficas vide Tabela 2. 


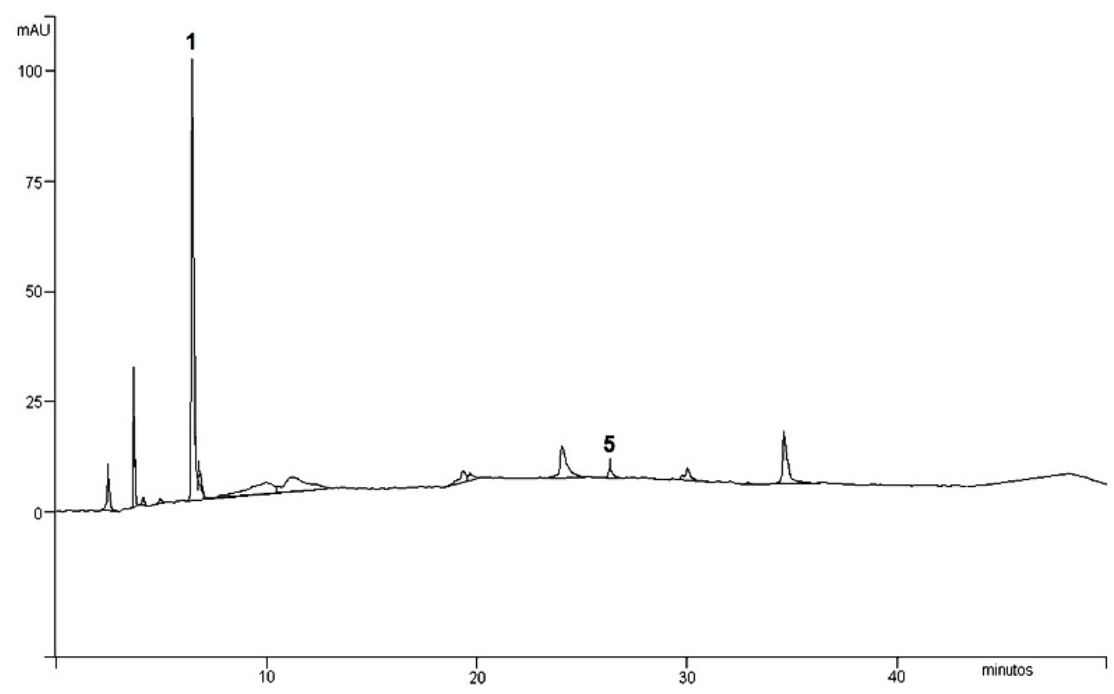

FIGURA 4. Perfil cromatográfico do extrato infuso das folhas secas de $C$. sessiliflora coletadas durante o período reprodutivo (RSIS). Condições cromatográficas vide Tabela 2.

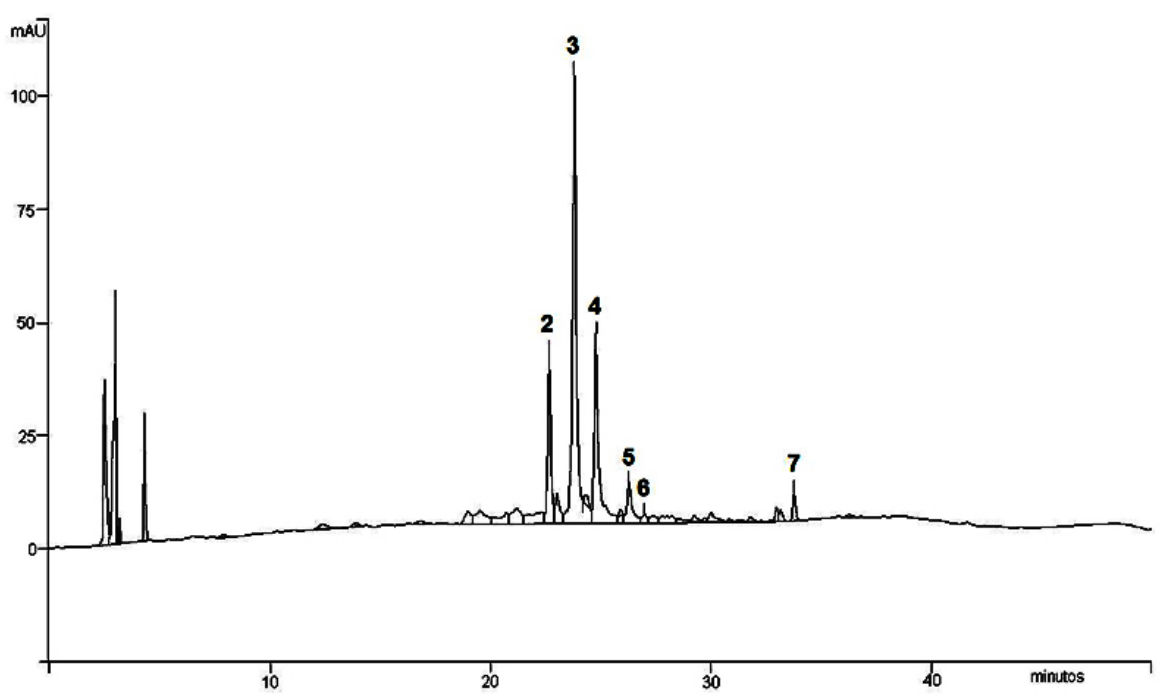

FIGURA 5. Perfil cromatográfico do extrato hidroalcóolico das folhas secas de $C$. xanthocarpa coletadas durante o período vegetativo (XE8S). Condições cromatográficas vide Tabela 2.

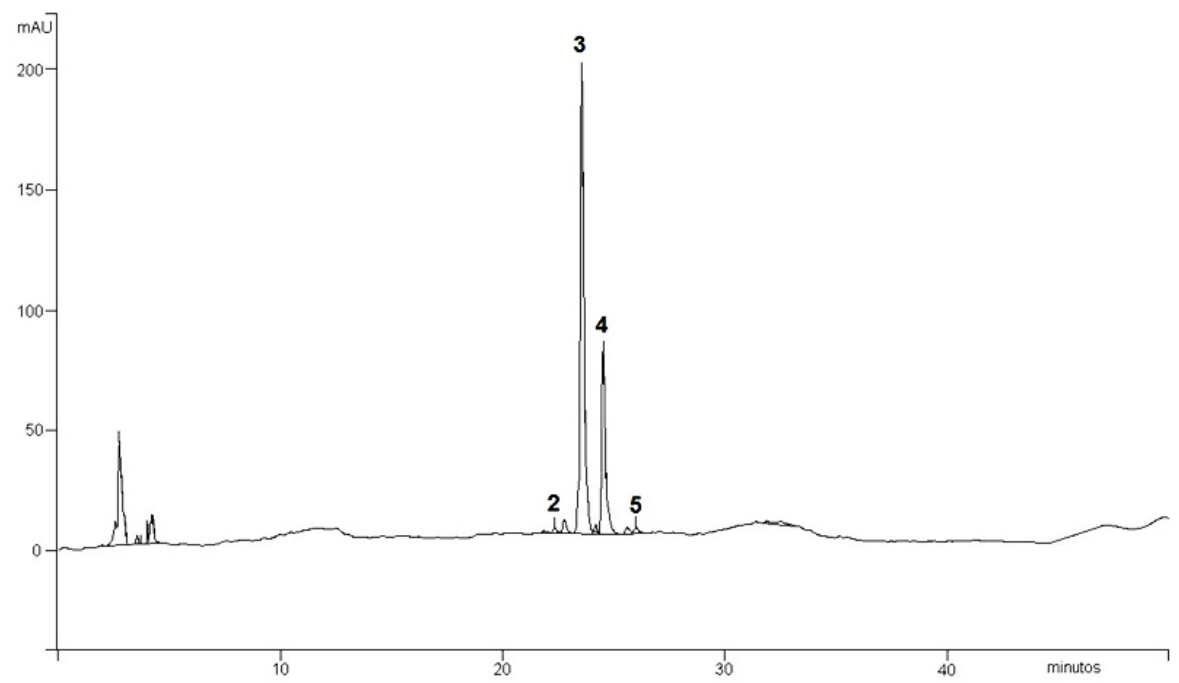

FIGURA 6. Perfil cromatográfico do extrato hidroalcóolico das folhas secas de C. xanthocarpa coletadas durante o período reprodutivo (RXE8S). Condições cromatográficas vide Tabela 2. 


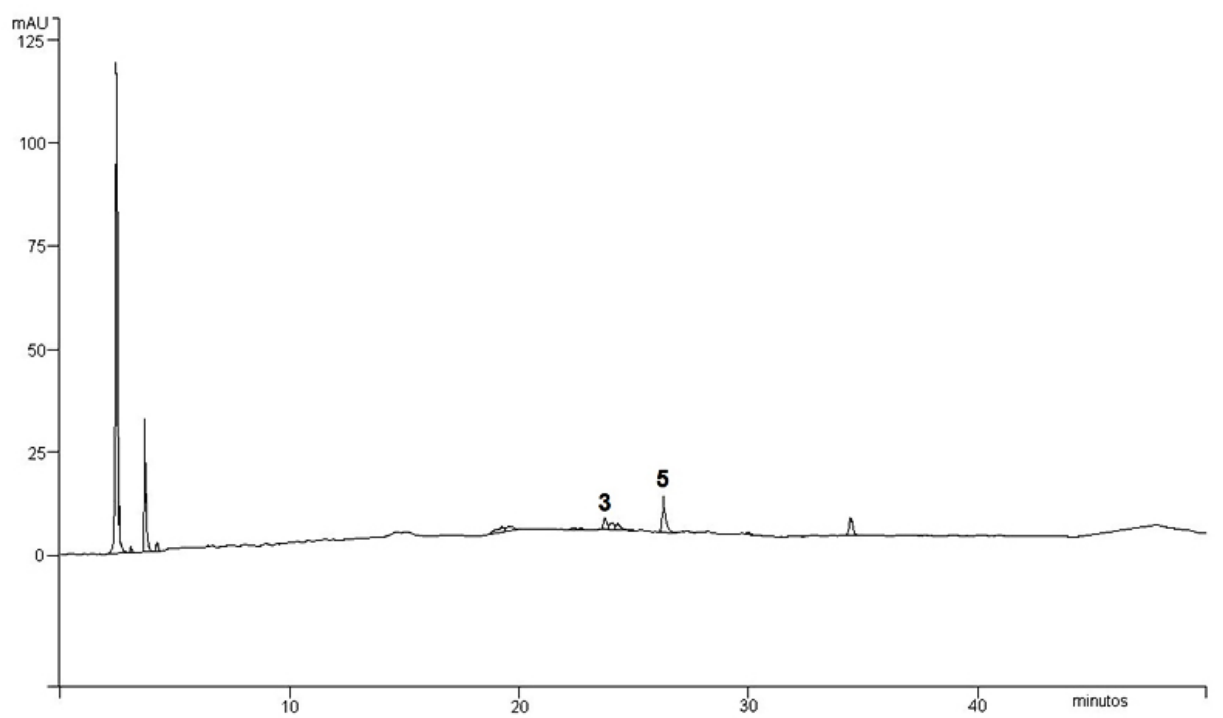

FIGURA 7. Perfil cromatográfico do extrato infuso das folhas secas de $C$. xanthocarpa coletadas durante 0 período vegetativo (XIS). Condições cromatográficas vide Tabela 2.

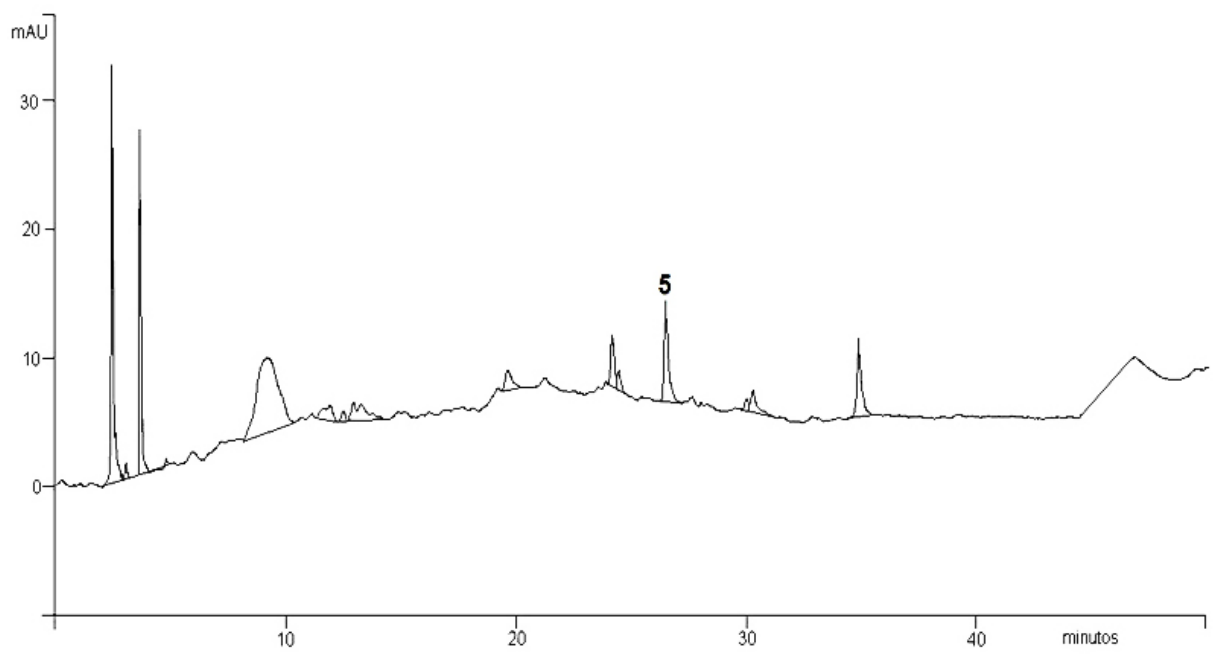

FIGURA 8. Perfil cromatográfico do extrato infuso das folhas secas de C. xanthocarpa coletadas durante o período reprodutivo (RXIS). Condições cromatográficas vide Tabela 2.

substâncias presentes nos extratos hidroetanólicos (Figuras 1, 2, 4 e 5). Os picos majoritários presentes nas análises dos extratos hidroalcoólicos não aparecem na infusão.

$\mathrm{Na}$ análise de fenóis totais e flavonóides o coeficiente de variação foi menor ou igual a $5 \%$ em todas as amostras. Os flavonóides são um grupo de compostos que pertencem à classe dos fenóis e em todas as amostras analisadas neste trabalho o teor de fenóis totais foi superior ao teor de flavonóides (Figura 9). Os teores de fenóis totais e flavonóides foram superiores para os extratos hidroetanólicos e infusos das folhas coletadas no período reprodutivo em ambas as espécies. Pelos espectros de absorção molecular os picos do estágio reprodutivo apresentam bandas características do sistema cinamoil do esqueleto de chalconas e do sistema benzoil do esqueleto de flavanonas (Lin \&
Tang, 2007) o que pode explicar o valor maior obtido no teste espectrofotométrico para esta classe de substâncias.

Dentre os picos monitorados (Tabela 3), os picos 3 e 4 apresentaram perfis de espectros semelhantes ao de flavanonas $\left(\lambda_{\text {Abs }}\right.$ de 286-291 $\mathrm{nm}$ ), e de acordo com a figura 9 , a presença destes picos pode ter contribuído de forma significativa para o teste de flavonóides e fenóis, já que estes picos foram encontrados nos extratos hidroetanólicos dos dois períodos para C. xanthocarpa, equilibrando a resposta, entretanto, a ausência do pico 4 pode ter influenciado na redução da resposta de XIS em relação a RXIS que não apresentou nenhum dos picos, o que reforça a hipótese de outros componentes da amostra estarem atuando na resposta espectrofotométrica da espécie e de $C$. sessiliflora. 


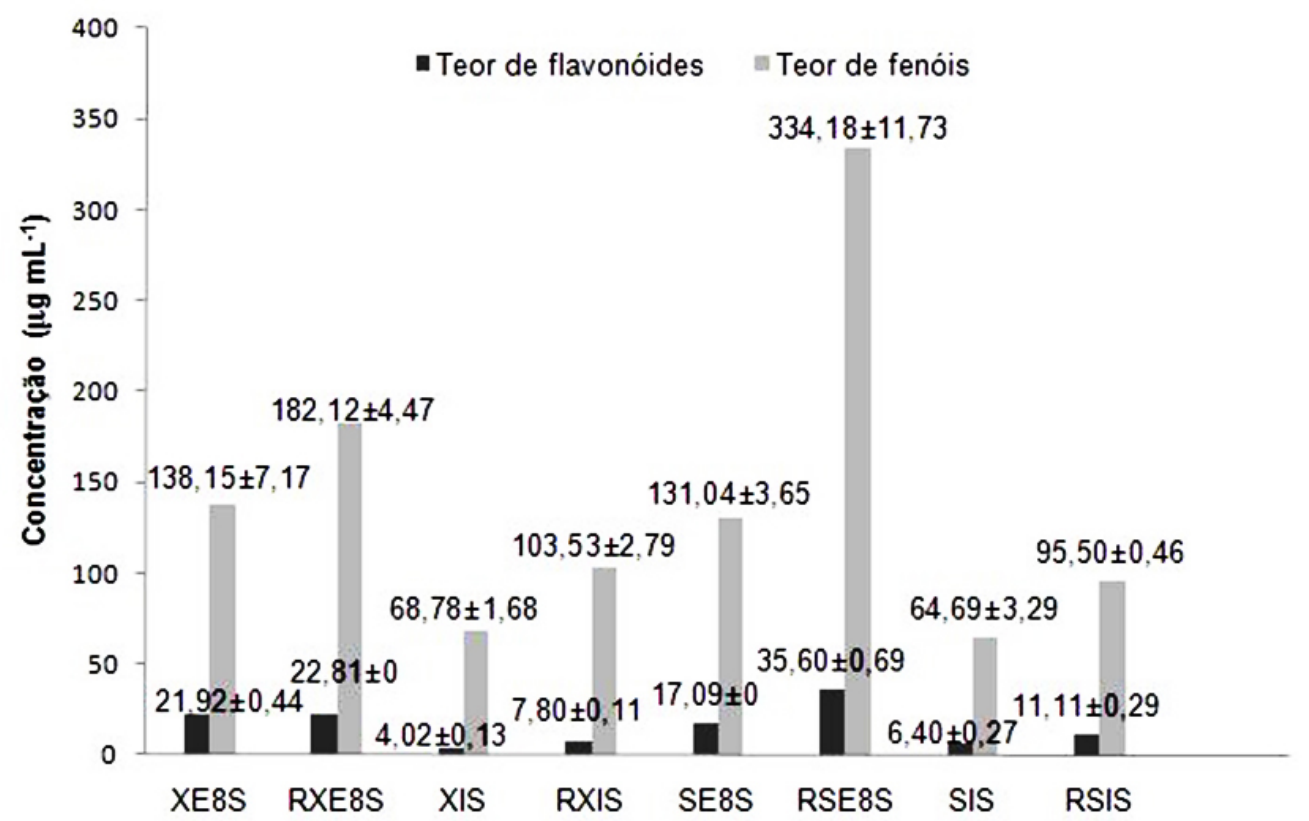

FIGURA 9. Teor de flavonóides ( $\mu \mathrm{g}$ de quercetina $\mathrm{mL}^{-1}$ ) e teor de fenóis ( $\mu \mathrm{g}$ de ácido gálico $\mathrm{mL}^{-1}$ ) obtidos em

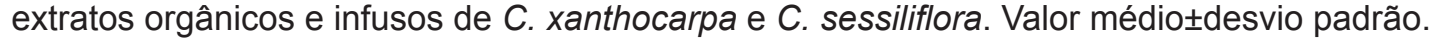

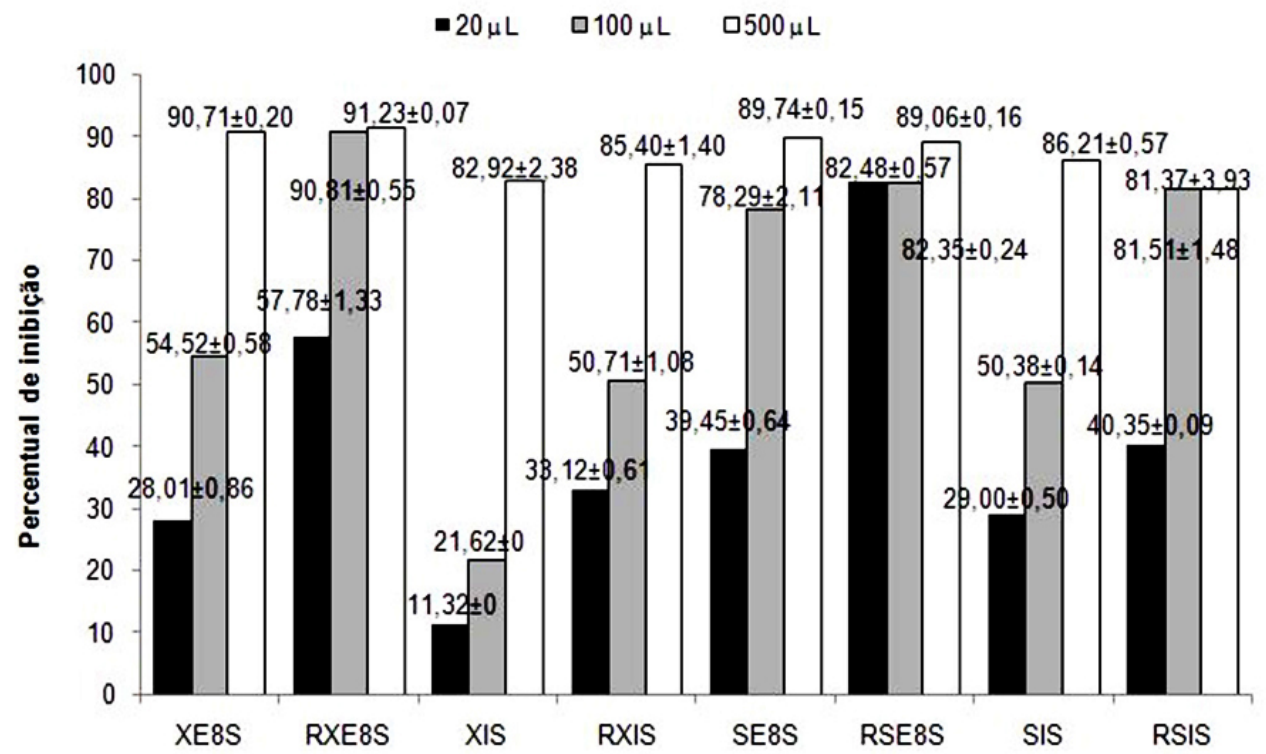

FIGURA 10. Percentual de inibição do radical livre DPPH obtido nas análises de C. xanthocarpa e C. sessiliflora. Valor médio \pm desvio padrão.

Para a atividade antioxidante o coeficiente de variação resultante foi menor que $5 \%$ em todas as análises. Na tentativa de correlacionar os resultados espectrofotométricos para cada amostra, pode-se concluir que não houve uma correlação linear entre o teor de fenóis e flavonóides com a atividade antioxidante para todas as amostras, isso porque para algumas amostras com maiores teores de fenóis e flavonóides, não foram obtidos os mais elevados valores de percentual de inibição.
Mesmo as amostras apresentando teores de fenóis e flavonóides variáveis a atividade antioxidante ficou praticamente inalterada em alta concentração em todas as amostras (Figura 10). Nas outras duas diluições testadas houve uma discrepância maior dos dados, mas também não houve relação linear com o resultado obtido para fenóis e flavonóides. Este fato deve ser ocorrido, pois nem todos os fenóis e flavonóides presentes nas amostras devem apresentar estruturas químicas que apresentem 
elevada atividade antioxidante empregando o DPPH. A atividade antioxidante depende do tipo de substituintes presentes nas estruturas básicas de fenóis e flavonóides, normalmente compostos com grupamentos polares, tais com hidroxilas, apresentam maior atividade antioxidante (Soares et al., 1997).

\section{AGRADECIMENTO}

À Fundação de Apoio ao Desenvolvimento do Ensino, Ciência e Tecnologia do Estado do Mato Grosso do Sul (Fundect) pelo apoio financeiro concedido a pesquisa, UEMS e CNPq.

\section{REFERÊNCIA}

BIAVATTI, M.W. et al. Preliminary studies on Campomanesia xanthocarpa and Cuphea carthagenensis (Jacq.) J. F. Macbr. aqueous extract: Weight control and biochemical parameters. Journal Ethnopharmacology, v.93, n.2-3, p.385-9, 2004.

CARDOSO, C.A.L. et al. Fruit oil of Campomanesia xantocarpa O. Berg and Campomanesia adamantium O.Berg. Journal of Essential Oil Research, v.21, p.4813, 2009.

DJERIDANE, A. et al. Antioxidant activity of some Algerian medicinal plants extracts containing phenolic compounds. Food Chemistry, v.97, n.4, p.654-60, 2006.

GNOATTO, M. et al. Caracterização do óleo essencial das folhas de Campomanesia sessiliflora e Campomanesia guaviroba empregando CG-EM. In: REUNIÃO ANUAL DA SOCIEDADE BRASILEIRA DE QUÍMICA, 33., 2010, Águas de Lindóia. Resumos... Águas de Lindóia: Sociedade Brasileira de Química, 2010.

KLAFKE, J.Z. et al. The Effect Of Campomanesia xanthocarpa "Guabiroba" In Hypercholesterolemic Subjects. Circulation, v.118, p.382, 2008.

KLAFKE, J.Z. et al. Effects of Campomanesia xanthocarpa on biochemical, hematological and oxidative stress parameters in hypercholesterolemic patients. Journal of Ethnopharmacology, v.127, p.299-305, 2010.

KUMARAN A.; KARUNAKARAN, R.J. Antioxidant and free radical scavenging activity of an aqueous extract of Coleus aromaticus. Food Chemistry, v.97, n.1, p.109-14, 2006. LIMBERGER, R.P. et al. Aromatic plant from Brazilchemical composition of essencial oils from some Campomanesia species (Myrtaceae). The Journal of Essential Oil Research, v.13, p.113-5, 2001.

LIN, J-Y.; TANG, C-Y. Determination of total phenolic and flavonoid contents in selected fruits and vegetables, as well as their stimulatory effects on mouse splenocyte ploriferation. Food Chemistry, v.101, n.1, p.140-7, 2007. LORENZI, $\mathrm{H}$. et al. Frutas brasileiras e exóticas cultivadas (de consumo in natura). Nova Odessa: Instituto Plantarum de Estudos da Flora Ltda, 2006. p.178-90.

MARIN, R. et al. Volatile Components and Antioxidant Activity from some Myrtaceous Fruits cultivated in Southern Brazil. Latin American Journal of Pharmacy, v.27, n.2, p.172-7, 2008.

MARKMAN, B.E.O. Caracterização farmacognóstica de Campomanesia xanthocarpa Berg Myrtaceae. 2002. 169p. Dissertação (Mestrado - Área de Concentração em Insumos Farmacêuticos) - Faculdade de Ciências Farmacêuticas, Universidade de São Paulo, São Paulo. MARKMAN, B.E.O. et al. Atividade antimicrobiana do extrato hidroalcoolico de Campomanesia xanthocarpa. Revista Brasileira de Ciências Farmacêuticas, v.36, p.55, 2002.

MARKMAN, B.E.O.; BACCHI, E.M.; KATO, E.T.M. Antiulcerogenic effects of Campomanesia xanthocarpa. Journal of Ethnopharmacology, v.94, n.1, p.55-7, 2004. MENUT, C. et al. Chemical exploration of brazilian aromatic species belonging to the Myrtaceae family. In: INTERNATIONAL SYMPOSIUM ON ESSENTIAL OILS, 27., 1996, Vienna. Proceedings... Vienna: Allured Publishing Corporation, 362 South Schmale Rd., Carol Stream, IL 60188-2787, USA, 1996. p.171-83.

SANTOS, M.S. et al. Caracterização físico-química, extração e análise de pectinas de frutos de Campomanesia xantthocarpa B. Ciências de Alimentos, v.30, n.1, p.1016, 2009.

SANTOS, M.S. et al. Polissacarídeos Extraídos da Gabiroba (Campomanesia xanthocarpa Berg): Propriedades Químicas e Perfil Reológico. Polímeros, v.20, p.352-8, 2010.

SANTOS, M.S. et al. Antioxidant and fatty acid profile of gabiroba seed (Campomanesisa Xanthocarpa Berg). Ciência e Tecnologia de Alimentos, v.32, n.2, p.234-8, 2012.

SCHMEDA-HIRSCHMANN, G. Flavonoids from Calycorectes, Campomanesia, Eugenia and Hexachlamys species. Fitoterapia, v.66, n.4, p.373-4, 1995.

SOARES, J.R. et al. Antioxidant activities of some extracts of Thymus zygis. Free Radical Research, v.26, p.46978, 1997

VALLILO, M.I. et al. Composição química dos frutos de Campomanesia xanthocarpa Berg-Myrtaceae. Ciência e Tecnologia de Alimentos, supl.28, p.231-7, 2008.

VINAGRE, A.S. et al. Anti-diabetic effects of Campomanesia xanthocarpa (Berg) leaf decoction. Brazilian Journal of Pharmaceutical Sciences, v.46, n.2, p.169-77, 2010.

Rev. Bras. PI. Med., Botucatu, v.15, n.1, p.121-129, 2013. 